\title{
Simulation of Human Phonation with Vocal Nodules
}

\author{
Shinji Deguchi, ${ }^{1,2, *}$, Yuki Kawahara ${ }^{3}$ \\ ${ }^{1}$ Department of Biomedical Engineering, Tohoku University, Sendai, Japan \\ ${ }^{2}$ Department of Bioengineering and Robotics, Tohoku University, Sendai, Japan \\ ${ }^{3}$ Graduate School of Natural Science and Technology, Okayama University, Sendai, Japan \\ E-mail:"deguchi@bml.mech.tohoku.ac.jp \\ Received June 16, 2011; revised July 20, 2011; accepted August 2, 2011
}

\begin{abstract}
The geometric and biomechanical properties of the larynx strongly influence voice quality and efficiency. A physical understanding of phonation natures in pathological conditions is important for predictions of how voice disorders can be treated using therapy and rehabilitation. Here, we present a continuum-based numerical model of phonation that considers complex fluid-structure interactions occurring in the airway. This model considers a three-dimensional geometry of vocal folds, muscle contractions, and viscoelastic properties to provide a realistic framework of phonation. The vocal fold motion is coupled to an unsteady compressible respiratory flow, allowing numerical simulations of normal and diseased phonations to derive clear relationships between actual laryngeal structures and model parameters such as muscle activity. As a pilot analysis of diseased phonation, we model vocal nodules, the mass lesions that can appear bilaterally on both sides of the vocal folds. Comparison of simulations with and without the nodules demonstrates how the lesions affect vocal fold motion, consequently restricting voice quality. Furthermore, we found that the minimum lung pressure required for voice production increases as nodules move closer to the center of the vocal fold. Thus, simulations using the developed model may provide essential insight into complex phonation phenomena and further elucidate the etiologic mechanisms of voice disorders.
\end{abstract}

Keywords: Aerodynamics, Flow-Structure Interaction, Self-Excited Oscillation, Phonation, Vocal Fold, Larynx, Vocal Tract, Speech Production, Voice Production, Vocal Nodules, Vocal Cord Polyp, Voice Disorder

\section{Introduction}

Human phonation consists of the following mechanical processes: 1) induction of self-excited vocal fold oscillations by respiratory airflow through flow-structure interaction, 2) acoustic resonance of sounds generated by the oscillations in the airway, i.e., the passage from the lungs to the mouth and nose, and 3) emission of the sounds to the surrounding atmosphere as voice. Mathematical modeling and computer simulation of phonation, thus a complicated mechanical phenomenon, may be useful for future clinical diagnosis and therapy of diseased or injured vocal folds.

Various numerical models have been reported for phonation simulation. One of the earliest was provided by Ishizaka and Flanagan [1], in which the vocal folds were approximated by two connected masses supported by nonlinear springs and dampers. In addition to flow- induced self-excitation of the vocal folds as a source of voice sound, their model considered virtually all mechanical phenomena occurring during phonation, including sound resonance in the vocal tract (i.e., the pharynx and the oral and nasal cavities) and sound emission into the atmosphere. However, structural and histological details for the vocal folds were not incorporated in their lumped two-mass model. To identify the etiology of voice disorders based on such simulations, vocal fold models are required to capture relevant anatomical and functional characteristics [2]. Story and Titze [3] proposed an alternative vocal fold model consisting of three masses, i.e., a deep mass representing the body of the vocal fold (modeling the thyroarytenoid (TA) muscle and the deep layer of the lamina propria) and the other two masses representing the cover of the vocal fold (modeling the epithelium as well as the superficial and intermediate layers of the lamina propria). Two-dimensional vocal fold 
shapes were considered in the model, and vocal fold movement in the anterior-posterior plane was ignored. Many other two-dimensional models have been reported for use in phonation analysis [4-8]; however, these may be inadequate to deal with voice disorders caused by mass lesions localized in the anterior-posterior plane such as vocal nodules and polyps [9,10]. Some vocal fold models considering a three-dimensional structure have also been developed with analysis of fluid-induced selfexcited oscillation [11-14]. However, previous studies typically used a quasi-steady Bernoulli flow model, whose application is limited at high voice fundamental frequencies $[15,16]$.

To our knowledge, Wong et al. [11] is the only numerical analysis that investigated the effect of an additional diseased mass localized in the anterior-posterior plane of the vocal folds. They investigated some pathological cases using a lumped-element model while changing the value of a local mass placed on the edge of the vocal fold. However, they did not examine possible changes in phonation threshold conditions and the fundamental frequency due to the additional mass. Thus, despite extensive research on phonation simulation, the dynamics of pathological vocal fold oscillation with mass lesions are still poorly understood.

In the present study, we build a phonation model that treats the vocal folds as three-dimensional shapes. The model is an extension of a previously reported approach $[4,6]$, in which the airway from the lungs to the mouth was considered together with vocal folds and approximated as a flexible channel coupled to an unsteady, compressible, and viscous flow allowing flow-structure interaction. Here, we expand upon the previous work by incorporating the mechanical properties reported of each of the three main layers that constitute the vocal folds, i.e., the mucosa, ligament, and muscle [2,3,17-21] for deriving motion equations. Additionally, phonation simulation is performed with or without bilateral masses added to the edge of the vocal folds, mimicking the vocal nodules. These results show that the additional massinduced reductions in the voice fundamental frequency and phonation threshold pressure are largest when the masses are at the center of the vocal folds in the anterior-posterior plane. The present pilot study may provide a basis for future simulation-based diagnosis of voice disorders.

\section{Methods}

\subsection{Lungs, Trachea, and Vocal Tract}

Numerical analyses were performed based on a model that includes the airway from the lungs to the mouth
(Figure 1). All modeled parts except for the vocal folds are the same as those used in our former studies $[4,6]$. Extensive modifications were made to the vocal folds as described in Section 2.2. The flow in the airway, described in detail in Section 2.3, was approximated to be one-dimensional. The $\mathrm{X}$-axis corresponds to the mainstream direction. The lungs were represented by a pressurized tank. The bronchi and trachea were approximated by a rigid pipe of a longitudinal length of $0.23 \mathrm{~m}$ with a uniform cross-sectional area of $2.45 \times 10^{-4} \mathrm{~m}^{2}$, a typical size for human adults. The vocal tract composed of the pharynx and oral cavity were represented by a rigid tube with a longitudinal length of $0.16 \mathrm{~m}$, the cross-sectional area of which varies along the flow direction to satisfy the resonance characteristics of the vowel /a:/ [22] (Figure 2).

\subsection{Vocal Fold Model}

The vocal folds were approximated as a viscoelastic membrane (Figure 3). The $X$-, $Y$-, and $Z$-axes indicate the directions of the thickness (equal to the flow direction; 0 $\leq X \leq 10.4 \mathrm{~mm}$ for the vocal folds), length $\left(0 \leq Y \leq l_{g}\right.$ where $l_{g}$ is the vocal fold length and is as $\left.14 \mathrm{~mm}\right)$, and depth of the vocal folds $(Z)$, respectively. Elements in the vocal folds were assumed to displace in the $Z$-axis perpendicular to the flow direction. Each individual fold was assumed to deform symmetrical to the medial axis $(Z=0)$. The three-dimensional prephonatory shape $B_{0}$ is described by

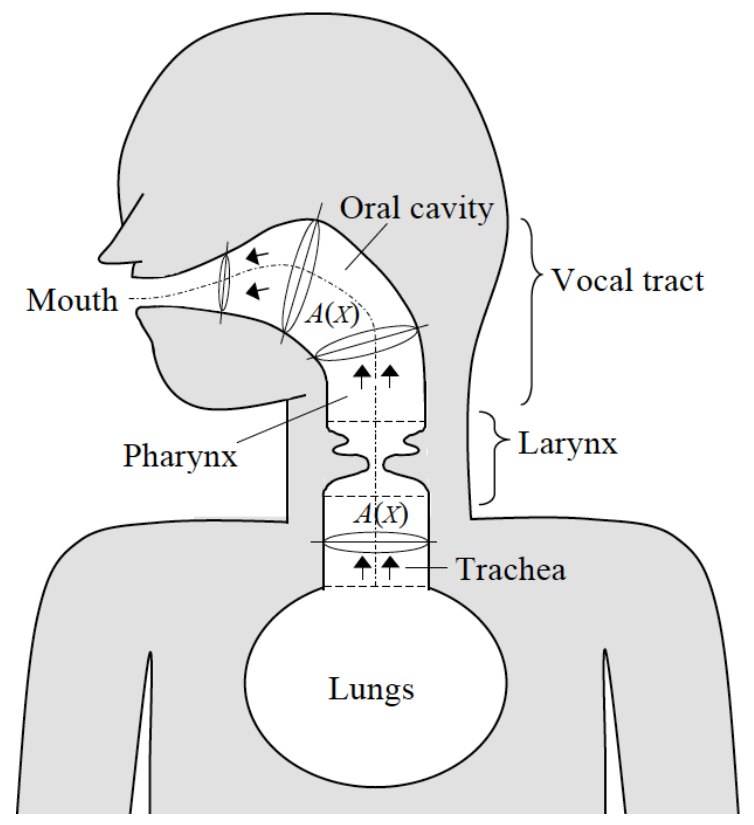

Figure 1. Schematic of the airway model. A frontal section is shown for the larynx, while sagittal sections are shown for other flow paths. 


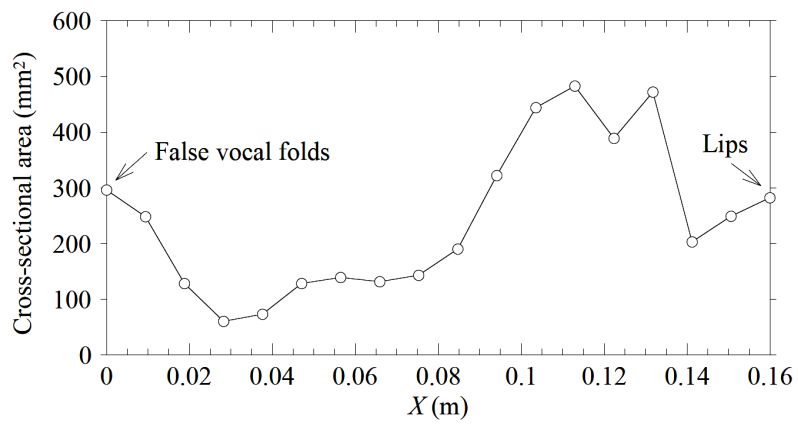

Figure 2. Cross-sectional area distribution of the vocal tract for the vowel /a:/. The horizontal axis is the distance from the upstream end of the vocal tract to the end of the larynx or false vocal folds (see Figure 3).

$$
\begin{aligned}
B_{0}= & \frac{2\left(B_{e n}-B_{e x}\right)-10.4 \cdot \tan 49^{\circ}}{10.4^{3}} X^{3} \\
& +\frac{3\left(B_{e n}-B_{e x}\right)+2 \cdot 10.4 \cdot \tan 49^{\circ}}{10.4^{2}} X^{2} \\
& -X \tan 49^{\circ}+B_{e n}(\mathrm{~mm}), \\
B_{e x}= & B_{v p}\left(1-Y / l_{g}\right),
\end{aligned}
$$

where $B_{e n}$ (fixed to be $8.2 \mathrm{~mm}$ ) and $B_{e x}$ are the half-glottal widths at the entrance $(X=0 \mathrm{~mm})$ and exit $(X=10.4$ $\mathrm{mm})$, respectively, and $B_{v p}$ is the half-glottal width at the tip of the vocal process of the arytenoid cartilages ( $X=$ $10.4 \mathrm{~mm} ; Y=0 \mathrm{~mm}$ ) (Figure 3). Even when $B_{v p}$ is $0 \mathrm{~mm}$, it is assumed that the glottis has a small rear gap (posterior glottis) between the separate arytenoid cartilages that do not vibrate during phonation. The prephonatory shape within the $X-Z$ plane is described in detail in previous studies [6].

Following the body-cover theory $[17,19]$, the vocal folds are assumed to consist of three layers, the mucosa, ligament, and TA muscle. The mucosa, representing the epithelium and superficial layer of the lamina propria, covers the vocal folds. The vocal ligament, underlying the mucosa and overlying the muscle, consists of intermediate and deep layers of the lamina propria. It has an anterior-posterior orientation between the anterior commissure $\left(Y=l_{\mathrm{g}}\right)$ and vocal processes $(Y=0 \mathrm{~cm})$. The TA muscle is juxtaposed to the ligament. It is assumed that no relative displacement occurs between the interfaces of each layer. The equation of motion is derived considering the force balance for an infinitesimal element in the vocal fold (Figure 3) given as

$$
\begin{aligned}
& \rho_{v} h \frac{\partial^{2} B}{\partial T^{2}}+F_{c o l}=P+\frac{\partial\left(T_{x} \sin \theta_{x}\right)}{\partial X}+\frac{\partial\left(T_{y} \sin \theta_{y}\right)}{\partial Y}, \\
& \sin \theta_{x}=\frac{\partial B}{\partial X} / \sqrt{1+\left(\frac{\partial B}{\partial X}\right)^{2}},
\end{aligned}
$$

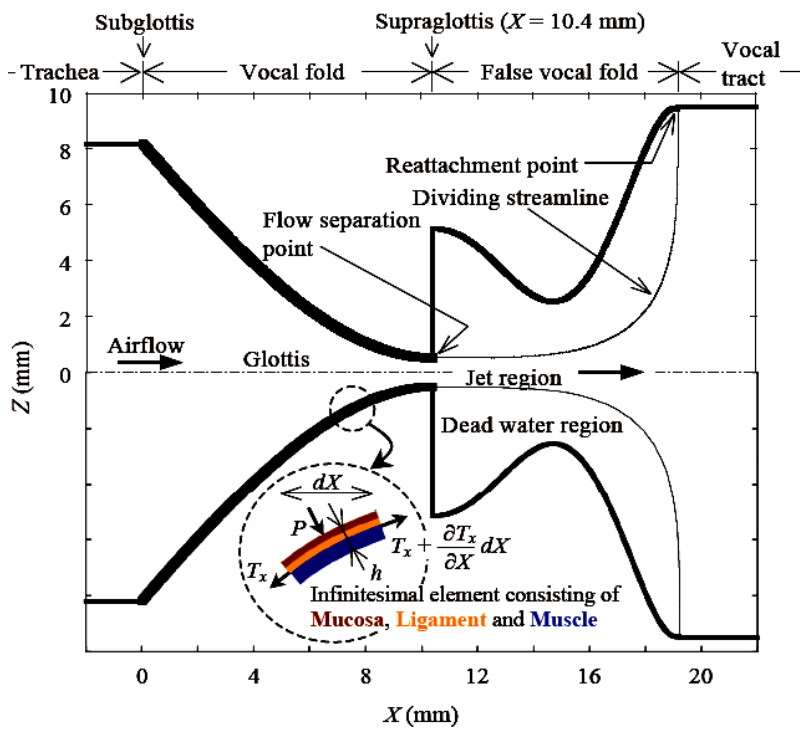

(a)

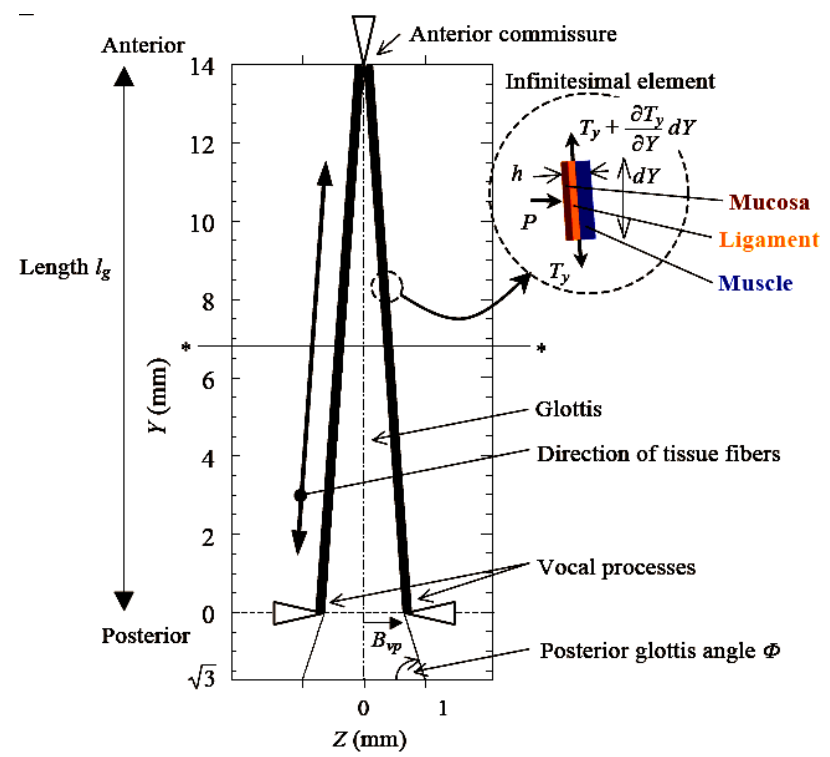

(b)

Figure 3. Schematic of the larynx model. (a) Initial geometry of vocal fold model in the coronal $(X-Z)$ plane. (b) Upper view ( $Y-Z$ plane) of the vocal folds. Open triangles represent the tips of the vocal process of arytenoid muscle and anterior commissure where no displacement is permitted during oscillation. Asterisks represent the plane shown in (a).

$$
\sin \theta_{y}=\frac{\partial B}{\partial Y} / \sqrt{1+\left(\frac{\partial B}{\partial Y}\right)^{2}},
$$

where $\rho_{v}$ is the mass per unit volume, $h$ is the vocal fold depth effective for vibration, $B$ is the half-glottal width, $T$ is the time, $F_{c o l}$ is the collision force per unit area, $P$ is the static air pressure, $T_{x}$ and $T_{y}$ are tensions in the vocal folds in the $X$ - and $Y$-directions, respectively, and $\theta_{x}$ and $\theta_{y}$ are deformed angles of infinitesimal 
elements in the $X$ - and $Y$ - directions, respectively (Figure 3). Because limited data is available regarding the shear properties of each layer, for simplicity, tensional components of each layer are considered to calculate the restoring forces. The first term of the left side of Equation (3) represents the inertial force. The collision force of the second term occurs only when one of the vocal fold pair collides with the facing vocal fold pair at $Z=0$ (median line) and is described by

$$
F_{c o l}=K B+C \frac{\partial B}{\partial T}, \quad(\mathrm{~B} \leq 0),
$$

where $K$ and $C$ are the spring constant and the damping coefficient, respectively $[1,4,6]$. The first term of the right side of Equation (3) represents air pressure as determined from the flow equations described in Section 2.3 at each time step to allow fluid-structure interactions. The second and third terms on the right side of Equation (3) represent the viscoelastic restoring force contributed by the line tension in the $X$-direction $T_{x}$ and the line tension in the $Y$-direction $T_{\mathrm{y}}$, respectively. The tension in the $X$-direction is described by

$$
\begin{aligned}
& T_{x}=E_{x} h \hat{\varepsilon}_{x}+\eta_{x} h \frac{\partial \hat{\varepsilon}_{x}}{\partial T} \\
& \hat{\varepsilon}_{x}=\varepsilon_{x}\left(\varepsilon_{x 0}+1\right)+\varepsilon_{x 0} \\
& \varepsilon_{x}=\sqrt{1+\left(\frac{\partial B}{\partial X}\right)^{2}} / \sqrt{1+\left(\frac{\partial B_{0}}{\partial X}\right)^{2}}-1
\end{aligned}
$$

where $E_{x}$ is the Young's modulus of the vocal folds in the $X$-direction, $\hat{\varepsilon}_{x}$ is strain in the $X$-direction, $\eta_{x}$ is the damping coefficient in the $X$-direction, and $\varepsilon_{x 0}$ is the prestrain in the $X$-direction.

Before we explain the contents of $T_{y}$, which is the tension in the tissue fiber direction, we briefly review the structure-based function of the vocal folds. In vivo, the vocal folds change their length (in the anterior-posterior direction or $Y$-direction), thickness ( $X$-direction), and effective depth (Z-direction) with the activation of laryngeal muscles $[19,20]$. The major muscles associated with voice pitch regulation are the cricothyroid (CT) and the TA muscles. The CT muscle runs between the thyroid and cricoid cartilages, and its activation stretches the vocal folds, increasing tension. The TA muscle runs in juxtaposition of the vocal ligament and constitutes the vocal fold itself. Consequently, its contraction pulls the thyroid cartilage inward to reduce the length of the vocal folds. In prephonatory conditions, the TA muscle undergoes isometric contraction in which the vocal fold length is unchanged under a force balance in laryngeal muscles, while the TA muscle contraction contributes to a rise in the tension. To consider the structure and function, the contribution of stress in the TA muscle to $T_{y}$ is described by the sum of active and passive stress components, given as

$$
\sigma_{\text {muscle }}\left(\hat{\varepsilon}_{y}\right)=\sigma_{\text {passive }}\left(\hat{\varepsilon}_{y}\right)+\sigma_{\text {active }}\left(\hat{\varepsilon}_{y}\right),
$$

where the stresses are a function of strain in the $Y$ direction $\hat{\varepsilon}_{y}$ that is described by

$$
\hat{\varepsilon}_{y}=\left(\varepsilon_{y 0}+1\right) \frac{\sqrt{1+\left(\frac{\partial B}{\partial Y}\right)^{2}} \mathrm{~d} y}{\sqrt{l_{g}^{2}+B_{v p}^{2}}}-1,
$$

where $\varepsilon_{y 0}$ is the prestrain in the $Y$-direction associated with the activation of the CT muscle. We use a reported relationship between the stress and strain for the passive and active components defined as [23]:

$$
\begin{aligned}
& \sigma_{\text {passive }}\left(\hat{\varepsilon}_{y}\right)=\left\{\begin{array}{c}
0, \quad \hat{\varepsilon}_{y} \leq 0 \\
a_{1}\left(e^{b_{1} \hat{\varepsilon}_{y}}-1\right), \quad \hat{\varepsilon}_{y}>0
\end{array}\right. \\
& \sigma_{\text {active }}\left(\hat{\varepsilon}_{y}\right)=\left\{\begin{array}{c}
0, \quad \hat{\varepsilon}_{y} \leq-0.4 \\
a_{T A} c_{1}\left(\hat{\varepsilon}_{y}+0.4\right) e^{c_{2}\left(\hat{\varepsilon}_{y}+0.4\right)^{2}}, \quad \hat{\varepsilon}_{y}>-0.4,
\end{array}\right.
\end{aligned}
$$

where $a_{1}=1.5 \times 10^{4} \mathrm{dyn} / \mathrm{cm}^{2}, \quad b_{1}=8.0, c_{1}=$ $(100 \sqrt{e} / 0.6) \times 10^{4} \mathrm{dyn} / \mathrm{cm}^{2}$, and $c_{2}=-1 /\left(2 \times 0.6^{2}\right)$. These coefficients are determined from the literature [23]. The parameter $a_{T A}$ represents the extent of TA muscle activity that ranges from 0 (inactive) to 1 (full isometric contraction), following previous studies [13,19-21,23]. The effect of $a_{T A}$ is shown in Figure 4 in which the stress-strain curves of the $T A$ muscle used in the present analysis are plotted. The cover and the ligament are assumed to bear passive stresses of the following forms:

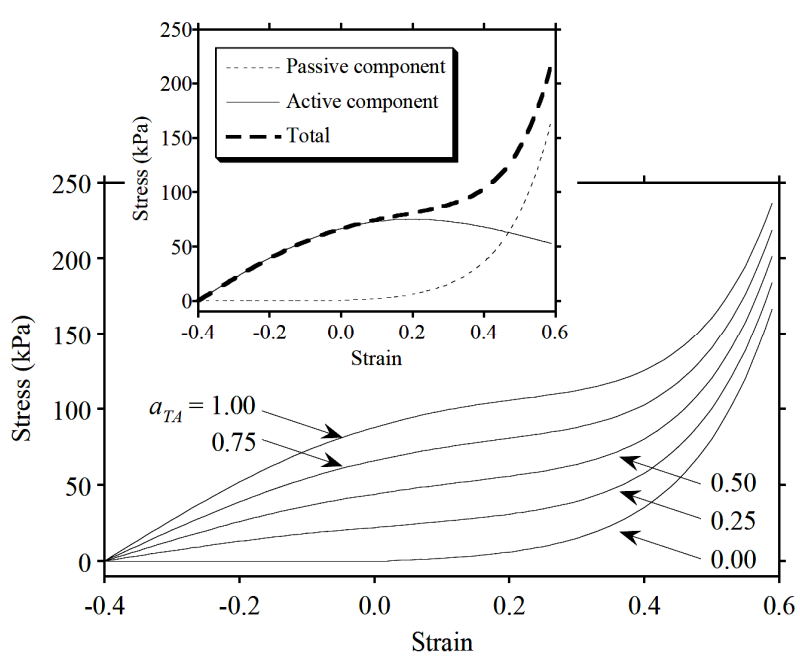

Figure 4. Stress-strain relationship of the thyroarytenoid muscle as a function of muscle activity $\mathbf{a}_{T A}$. Inset represents the sum of passive (Equation (12)) and active (Equation (13)) stress components described in Equation (10). 


$$
\begin{gathered}
\sigma_{\text {cover }}\left(\hat{\varepsilon}_{y}\right)=\left\{\begin{array}{c}
0, \quad\left(\hat{\varepsilon}_{y} \leq 0\right) \\
a_{2}\left(e^{b_{2} \hat{\varepsilon}_{y}}-1\right), \quad\left(\hat{\varepsilon}_{y}>0\right),
\end{array}\right. \\
\sigma_{\text {ligament }}\left(\hat{\varepsilon}_{y}\right)=\left\{\begin{array}{c}
0, \quad\left(\hat{\varepsilon}_{y} \leq 0\right) \\
a_{3}\left(e^{b_{3} \hat{\varepsilon}_{y}}-1\right), \quad\left(\hat{\varepsilon}_{y}>0\right),
\end{array}\right.
\end{gathered}
$$

where $a_{2}=7.1 \times 10^{4} \mathrm{dyn} / \mathrm{cm}^{2}, b_{2}=4.2, a_{3}=5.56 \times 10^{4}$ $\mathrm{dyn} / \mathrm{cm}^{2}$, and $b_{3}=5.15$. These stress-strain relationships are also based on data shown in the previous literature [23]. Consequently, the tension in the vocal folds obtained from the stresses in the three layers is given as

$$
\begin{aligned}
T_{y}= & h_{m} \sigma_{\text {muscle }}\left(\hat{\varepsilon}_{y}\right)+h_{l} \sigma_{\text {ligament }}\left(\hat{\varepsilon}_{y}\right) \\
& +h_{c} \sigma_{\text {cover }}\left(\hat{\varepsilon}_{y}\right)+\eta_{y} h \frac{\partial \hat{\varepsilon}_{y}}{\partial T},
\end{aligned}
$$

where $h_{m}, h_{l}$, and $h_{c}$ are the depths of the muscle, the ligament, and the cover layers, respectively, and $\eta_{y}$ is a damping coefficient. The last term represents tissue damping that is assumed to be proportional to the first time derivative of $\varepsilon_{y}$.

\subsection{Flow Model}

Airflow is described by the following equations of continuity and motion for a one-dimensional unsteady, compressible, and viscous flow $[4,6,15,16]$ :

$$
\begin{aligned}
& A \frac{\partial P}{\partial T}+Q \frac{\partial P}{\partial X}+\rho_{\mathrm{a}} a^{2}\left(\frac{\partial A}{\partial T}+\frac{\partial Q}{\partial X}\right)=0, \\
& \frac{\partial}{\partial T}\left(\frac{Q}{A}\right)+\frac{1}{2} \frac{\partial}{\partial X}\left(\frac{Q}{A}\right)^{2}+\frac{1}{\rho_{\mathrm{a}}} \frac{\partial P}{\partial X}+G=0,
\end{aligned}
$$

where $A, Q, \rho_{a}, a$, and $G$ are the cross-sectional areas of the airway, volume flow, air density, sound speed, and viscous terms, respectively. To evaluate pressure distribution along the false glottis, airflow is assumed to separate at the downstream end of the glottis, become a parallel side jet, and reattach at the downstream end of the false glottis [4,6,24] (Figure 3). The false glottis is assumed not to deform. The flow streamline after separation is assumed to have a quartic curve shape based on fluid mechanical experiments and analyses [24]. The Hagen-Poiseuille velocity distribution with kinematic viscosity $v$ is assumed only for evaluation of the viscous term $G$. Here, a rectangular geometry with a depth of $L$ (equal to the vocal fold length) is assumed for the cross-section of the glottis. For the other airway, circular cylindrical flow paths are assumed. Consequently,

$$
G=3 v \frac{Q}{2 L B^{3}}
$$

for the glottis $(L=14+3=17 \mathrm{~mm}$; Figure 3(b) $)$ and

$$
G=8 \pi v \frac{Q}{A^{2}}
$$

for the other flow paths.

\subsection{Simulation}

At the upstream end of the trachea, the total pressure is assumed to be equal to the lung pressure. Phonation is assumed to be initiated by increasing the lung pressure $P_{l}$ in the following manner $[4,6]$ :

$$
P_{l}=\left\{\begin{array}{c}
\frac{P_{l s}}{2}\left\{1-\cos \left(\frac{\pi T}{T_{p}}\right)\right\}, \quad\left(0 \leq T<T_{p}\right) \\
P_{l s}, \quad\left(T \geq T_{p}\right)
\end{array}\right.
$$

where $P_{l s}$ and $T_{p}$ are the final value of $P_{l}$ and the time required to achieve $P_{l s,}$, respectively. The pressure at the downstream end of the airway (i.e., the mouth) is given by the following equation that represents a radiation load on a circular piston in an infinite baffle [1]:

$$
P=\rho \frac{8}{3} \sqrt{\frac{A}{\pi^{3}}} \frac{\partial U}{\partial T} .
$$

It is assumed that no displacement of the vocal folds occurs at the anterior commissure $\left(Y=l_{\mathrm{g}}\right)$ and the arytenoid muscle ( $Y=-3 \mathrm{~mm}$ to $0 \mathrm{~mm}$ ) along the $X$-axis (Figure 3).

For phonation simulation with bilateral vocal nodules, an additional mass of $M_{V N}=0.15 \mathrm{~g}$ is added to each vocal fold pair. Unless otherwise stated, the bilateral masses are localized to a point at the supraglottis $(X=$ $10.4 \mathrm{~mm})$ close to the center of the vocal fold length $(Y=$ $6.4 \mathrm{~mm}$ ), i.e., the size of the vocal nodules is assumed to be zero in this case. To evaluate the effect of the localized mass, the mass is increased in value by a factor of 1.25 or 1.5. Alternatively, the additional masses placed at the supraglottis were distributed at either 1) $Y=6.4 \mathrm{~mm}$ and $Y=7.4 \mathrm{~mm}$ (assuming that nodule size $=1 \mathrm{~mm}$ ) or 2) $Y=5.4 \mathrm{~mm}, Y=6.4 \mathrm{~mm}$, and $Y=7.4 \mathrm{~mm}$ (assuming that nodule size $=2 \mathrm{~mm}$ ). It is likely that appearance of vocal nodules results in a localized change in stiffness as well as mass of the folds. In our preliminary analysis, we observed that a localized change in stiffness in the vocal fold model resulted in chaotic vocal fold motions and sound waveforms, which was not observed with isolated changes in mass. This behavior is consistent with our previous analysis using a two-dimensional model $[4,6]$, in which a change in stiffness sometimes results in a chaotic behavior. However, a thorough analysis is needed to understand the range of observed chaotic behavior, which will be a subject of future investigation. The present analysis, as a pilot study, only investigates the effect 
of the addition of localized masses in the three-dimensional geometry.

An implicit time-differencing scheme is applied [4] to integrate the flow equations, Equations (17) and (18). The flow equations are coupled to the motion equation, Equation (3). The time increment is chosen to be $1.0 \times$ $10^{-5} \mathrm{~s}$. Table 1 lists the values of other fixed parameters used throughout the simulation.

\section{Results}

\subsection{Wavelike Motion of the Vocal Fold Model Similar to the Actual Behavior}

Numerical simulation of normal human phonation without nodules was performed to evaluate the validity of our model. As the lung pressure increased according to Equation (1), a small-amplitude oscillatory motion arose in the vocal folds via the fluid-structure interaction. The small motion gradually grew larger and spread over the entire vocal folds. At a steady state of established limit cycles (Figure 5), the glottis opened at the upstream subglottis first and then at the downstream supraglottis. Similarly, closure first occurred upstream then downstream, consistent with the wavelike motion of actual vocal folds or a rubber-sheet model [25]. Pronounced collisions between the facing vocal folds occurred at the supraglottal region. The observation of the downstream end $\left(B_{e x}\right)$ from the top view ( $Y-Z$ plane) showed relative motion between the anterior and posterior regions. During the process of initial oscillation development, anterior and posterior parts of the vocal folds moved nearly in the same phase (figure not shown). However, anterior preceded the posterior in phase at later steady-state conditions (Figure 6).

\subsection{Effect of Adduction on Fundamental Frequency}

The observed phase lead probably resulted from the slightly abducted glottal geometry of the posterior region, causing it to undergo a relatively weak interaction with the fluid. This resulted in a delayed reaction to flow compared to the anterior narrow glottal width and strong flow-structure interaction. Therefore, we investigated the effect of laryngeal adduction by changing the posterior glottis angle $\Phi$ as follows (Figure 3(b)):

$$
B_{v p}=1-\sqrt{3} / \tan \Phi(\mathrm{mm})
$$

The fundamental frequency of the steady-state oscillation increased as the vocal folds were more adducted (decrease in $\Phi$ ) at any TA muscle activity $a_{T A}$ (Figure 7(a)) and lung pressure (Figures 7(b) and (c)). Increase in the fundamental frequency of the self-excited oscillations reflects closer interaction between the structure and the fluid [16], consistent with the above interpretation.

\subsection{Effect of Bilaterally Localized Masses on Phonation}

Next, we investigated how the presence of bilaterally localized masses, which approximate the vocal nodules, affected the fold motion. The oscillatory motion was delayed in phase locally around the place of nodules (Figure 8; arrowhead). Therefore, it appeared that the anterior part, the nodule, and the posterior part moved like three masses interconnected by springs. At the same time, the anterior and posterior parts had a smooth second mode deformation. Consequently, in the $Y-Z$ plane, the vocal folds behaved similar to both a three-mass model and a four-mass model. Indeed, total pressure waveform at the mouth, corresponding to the produced voice, had a relatively high intensity at the third and fourth harmonics (Figure 9). On the other hand, the sound waveform obtained from the smooth normal vocal fold oscillation without nodules predominantly peaked at lower frequency second harmonics.

Table 1. Fixed model parameters.

\begin{tabular}{|c|c|c|}
\hline Symbol & Meaning & Value \\
\hline$a$ & Sound speed & $3.5 \times 10^{2} \mathrm{~m} / \mathrm{s}^{\mathrm{a}}$ \\
\hline$C$ & Additional damping constant & $10^{3} \mathrm{Ns} / \mathrm{m}^{3 \mathrm{~b}}$ \\
\hline$E_{x}$ & Young's modulus ( $X$-axis) & $4.0 \times 10^{5} \mathrm{~N} / \mathrm{m}^{2 \mathrm{a}}$ \\
\hline$h$ & Vocal fold thickness & $2 \mathrm{~mm}^{\mathrm{d}}$ \\
\hline$h_{c}$ & Cover thickness & $0.35 \mathrm{~mm}^{\mathrm{e}}$ \\
\hline$h_{l}$ & Ligament thickness & $0.8 \mathrm{~mm}^{\mathrm{e}}$ \\
\hline$h_{m}$ & Muscle thickness & $0.85 \mathrm{~mm}^{\mathrm{e}}$ \\
\hline$K$ & Additional spring constant & $1.0 \times 10^{6} \mathrm{~N} / \mathrm{m}^{3 \mathrm{a}, \mathrm{c}}$ \\
\hline$T_{p}$ & Time constant in Equation (21) & $1.0 \times 10^{-2} \mathrm{~s}^{\mathrm{a}}$ \\
\hline$\varepsilon_{x 0}$ & Prestrain ( $X$-axis $)$ & $0.2^{\mathrm{f}}$ \\
\hline$\varepsilon_{y 0}$ & Prestrain ( $Y$-axis $)$ & $0.2^{\mathrm{f}}$ \\
\hline$\eta_{x}$ & Damping coefficient ( $X$-axis) & $10^{2} \mathrm{Ns} / \mathrm{m}^{2 \mathrm{~g}}$ \\
\hline$\eta_{y}$ & Damping coefficient ( $Y$-axis) & $10^{2} \mathrm{Ns} / \mathrm{m}^{2 \mathrm{~g}}$ \\
\hline$v$ & Viscosity & $1.7 \times 10^{-5} \mathrm{~m}^{2} / \mathrm{s}^{\mathrm{a}}$ \\
\hline$\rho_{a}$ & Air density & $1.1 \mathrm{~kg} / \mathrm{m}^{3 \mathrm{a}}$ \\
\hline$\rho_{v}$ & Vocal fold density & $1.03 \times 10^{3} \mathrm{~kg} / \mathrm{m}^{3 \mathrm{e}}$ \\
\hline
\end{tabular}

${ }^{\mathrm{a}}$ Ikeda et al., 2001. ' Estimated from $2(1+\zeta) \sqrt{\rho_{v} h K}=2(1+0.3) \sqrt{\rho_{v} h K}$ a,c

${ }^{\mathrm{c}}$ Ishizaka and Flanagan, 1972. ${ }^{\mathrm{d}} h=h_{c}+h_{l}+h_{m} .{ }^{\mathrm{e}}$ Story and Titze, 1995.

${ }^{\mathrm{f}}$ Deguchi and Kawahara, 2011. ${ }^{\mathrm{g}}$ Estimated from $l_{g} C$. 


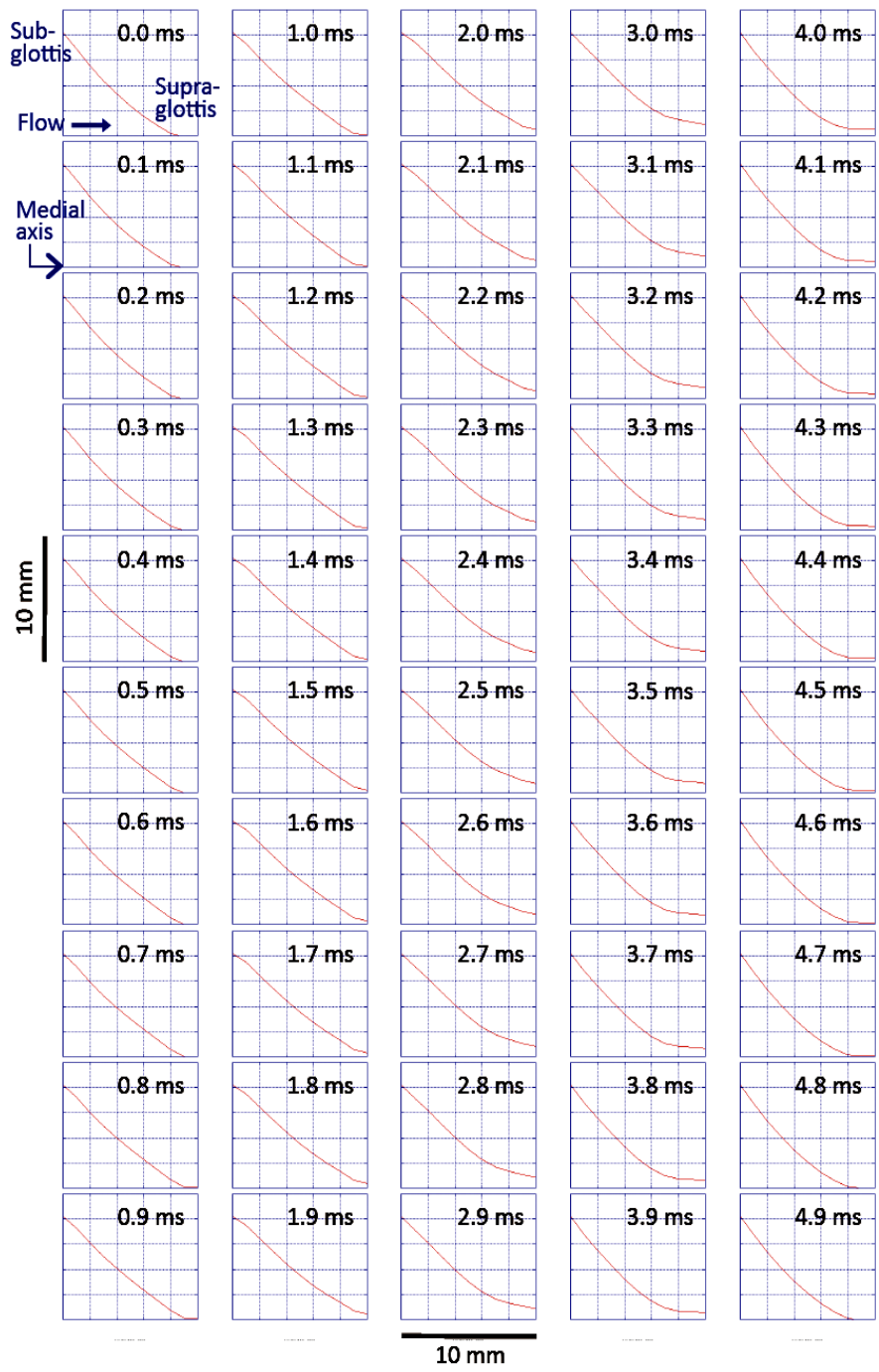

Figure 5. Sequential vocal fold deformation images in the coronal $(X-Z)$ plane during steady-state single cycle. Vocal fold shape and its motion were assumed to be symmetrical with respect to the midsagittal plane; for simplification, only one vocal fold is shown in this figure.

The fundamental frequency of the motion decreased as the vocal nodules increased in mass or size (Figure 10 (a)). This is consistent with the general tendency of oscillatory frequency to be inversely proportional to mass. The standard deviation of the total pressure at the mouth, corresponding to the loudness of the produced voice, also reduced similarly (Figure 10(b)) because the additional mass lowered the amplitude of the vocal fold motion. Overall, the additional mass and consequent decrease in amplitude prevented the fluid-structure interaction [15], resulting in decrease in volume flow (Figure 10(c)).

Phonation threshold pressure (PTP) is the minimum lung pressure required to initiate a vocal fold oscillation and has a potential diagnostic utility as a means of non-

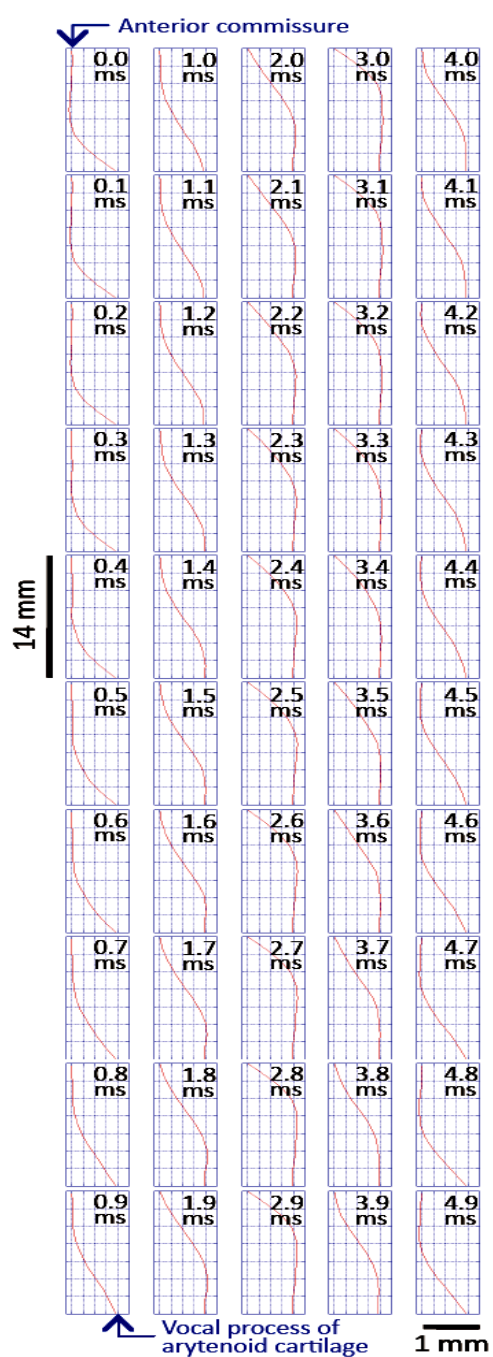

Figure 6. Sequential images of vocal fold deformation viewed from the top ( $Y-Z$ plane). The posterior glottis $(Y$ $=-3 \mathrm{~mm}$ to $0 \mathrm{~mm}$ ), which was assumed not to vibrate, is not shown. Note that the scale of the horizontal $(Z)$-axis is magnified to allow easier observation of the deformation.

invasively evaluating vocal fold stiffness and quantifying the ease of phonation [16,26,27]. We investigated how the position of the nodules in the anterior-posterior direction affects PTP. This analysis was carried out by dividing the vocal folds into ten sections along the $Y$-axis (vocal fold length $=14 \mathrm{~mm}$ ) and then changing the position of the nodules in $1.4 \mathrm{~mm}$ increments. PTP was determined at each nodule position by changing lung pressure till limit cycles developed. Limit cycles were obtained at a high lung pressure (Figure 11(a)) but not at a low lung pressure (Figure 11(b)). The critical lung pressure for the threshold was defined as PTP and was plotted as a function of nodule position (Figure 11(c)). With a fully adducted glottis $\left(\Phi=60^{\circ}\right)$, PTP had a maximum 


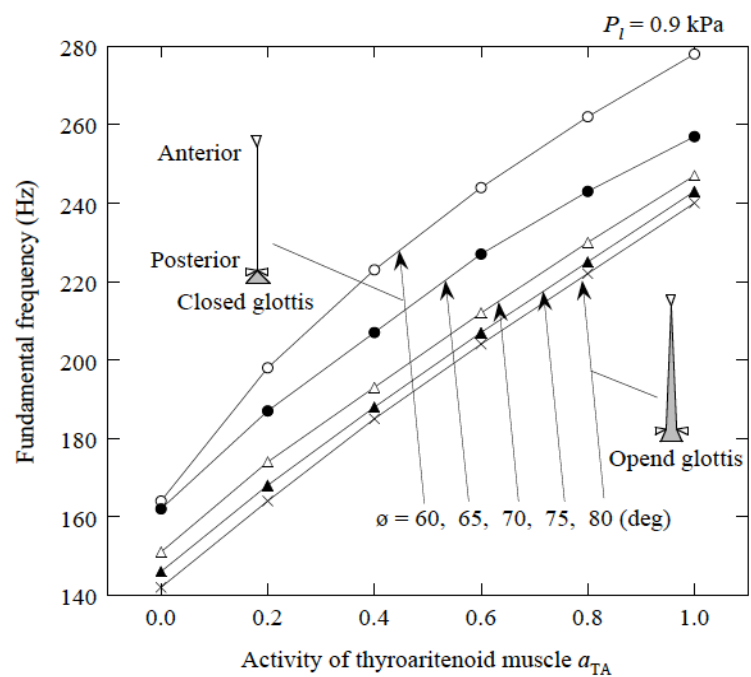

(a)

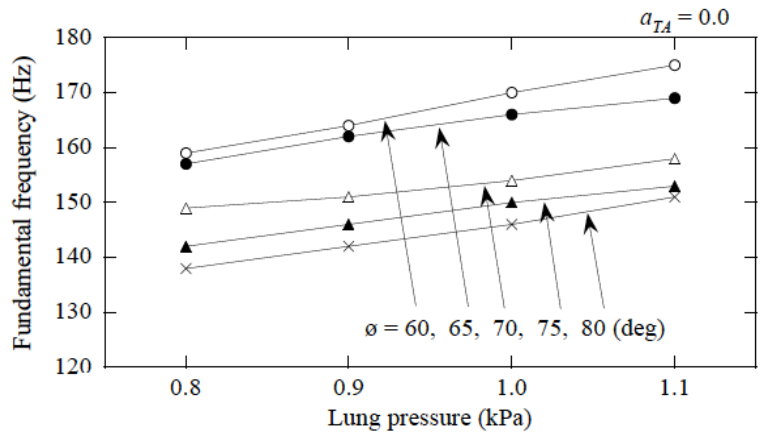

(b)

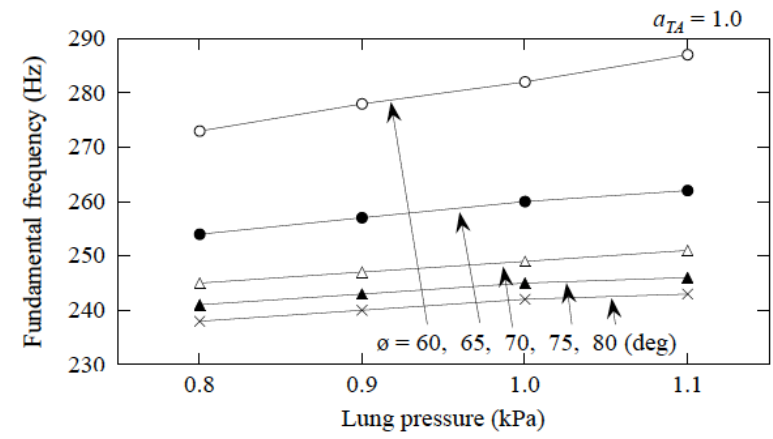

(c)

Figure 7. Effects of muscle activity, laryngeal adduction, and lung pressure on the fundamental frequency of voice. (a) Fundamental frequency vs. TA muscle activity $a_{T A}$ as a function of posterior glottis angle $\Phi$ at a lunge pressure $P_{I}$ of $0.9 \mathrm{kPa}$. (b) Fundamental frequency vs. $P_{l}$ as a function of $\Phi$ at an $a_{T A}$ of 0.0 . (c) Fundamental frequency vs. $P_{l}$ as a function of $\Phi$ at an $a_{T A}$ of 1.0.

value when the nodules were at the center of the vocal fold length, with a symmetrical curve to the center. PTP increased more than two-fold with nodules at the center $(0.92 \mathrm{kPa})$ than those at the edge $(0.44 \mathrm{kPa})$, indicating that phonation is more difficult when the vocal nodules

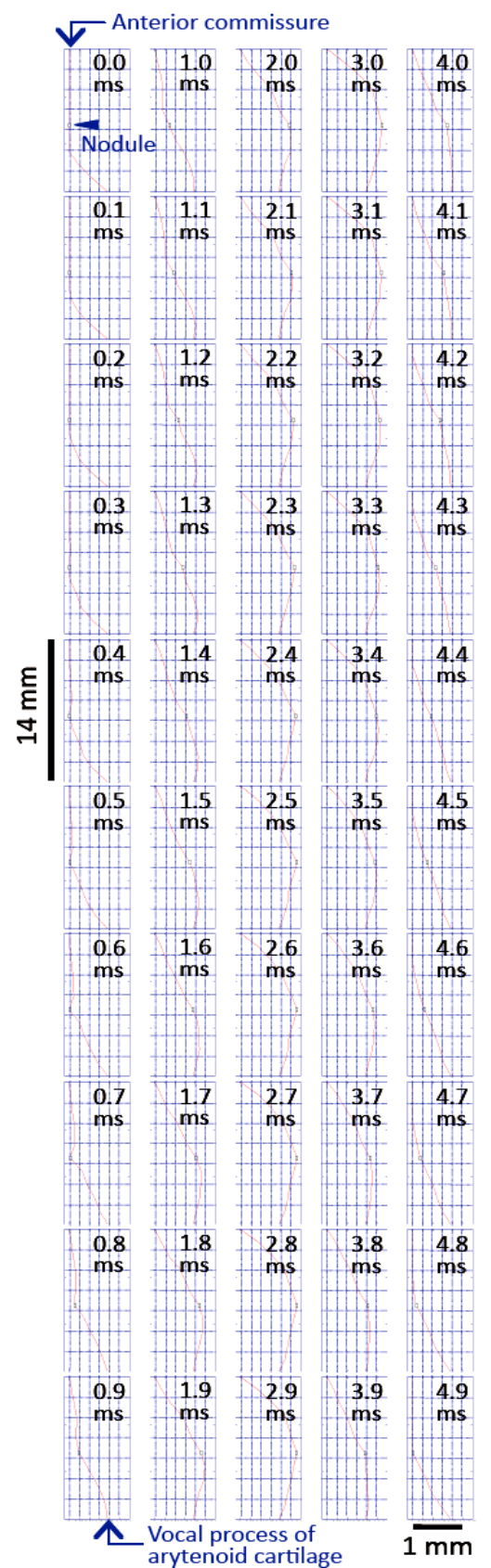

Figure 8. Oscillatory pattern of vocal folds with nodules (arrow) viewed from the top ( $Y-Z$ plane).

are closer to the fold center. The fundamental frequency of the limit cycles at each PTP also had a symmetrical curve with respect to the center, with $212 \mathrm{kPa}$ at the edge and $202 \mathrm{kPa}$ at the center (Figure 11(d)).

\section{Discussion}

To our knowledge, there has been no attempt to investigate how diseased vocal fold tissue affects voice production using a continuum mechanical model. It has been 
reported that the appearance of vocal nodules results in a localized change in mass and material properties such as the elastic modulus and the damping coefficient [9]. In a preliminary analysis, we noted that including localized change in the material properties of the present model often results in a chaotic vocal fold motion and sound waveforms, while a localized change in mass does not. Chaotic behavior is of interest in understanding voice disorder. However, given that material properties consist of multiple parameters in our three-dimensional model described in Section 2.2, thorough investigations on the effects of parameter variation required a fairly laborious work. In addition, it is poorly understood how material properties are altered by diseases. Currently, we presented results only pertaining to the effect of varying localized masses just to show our structurally and functionally adequate numerical model that allows the flowstructure-acoustic interaction throughout the airway. Our future directions include analysis of localized material property changes and more complicated geometrical de- generation such as bilateral swellings.

In the present analysis, the addition of a mass that approximates vocal nodules not only lowered the fundamental frequency of voice but also affected its harmonic components (Figures 8-10). We observed a marked increase in sound intensity at the third and fourth harmonics (Figure 9) after incorporation of the mass, originating from the awkward vocal fold motion (Figure 8). This is consistent with Hirose's monographic report on diseased oscillations [9], which demonstrates that anterior and posterior parts of the folds could oscillate across the nodules in an independent phase. Each part predominantly exhibited second mode oscillation in addition to first mode, thereby enhancing the fourth mode oscillation for both vocal folds. Hirose [9] also described that the vocal nodules could move in a different phase than the anterior and/or posterior parts, suggesting simultaneous presence of a three-mass mode oscillation. Thus, the presence of the additional mass could enhance higher order mode oscillations.

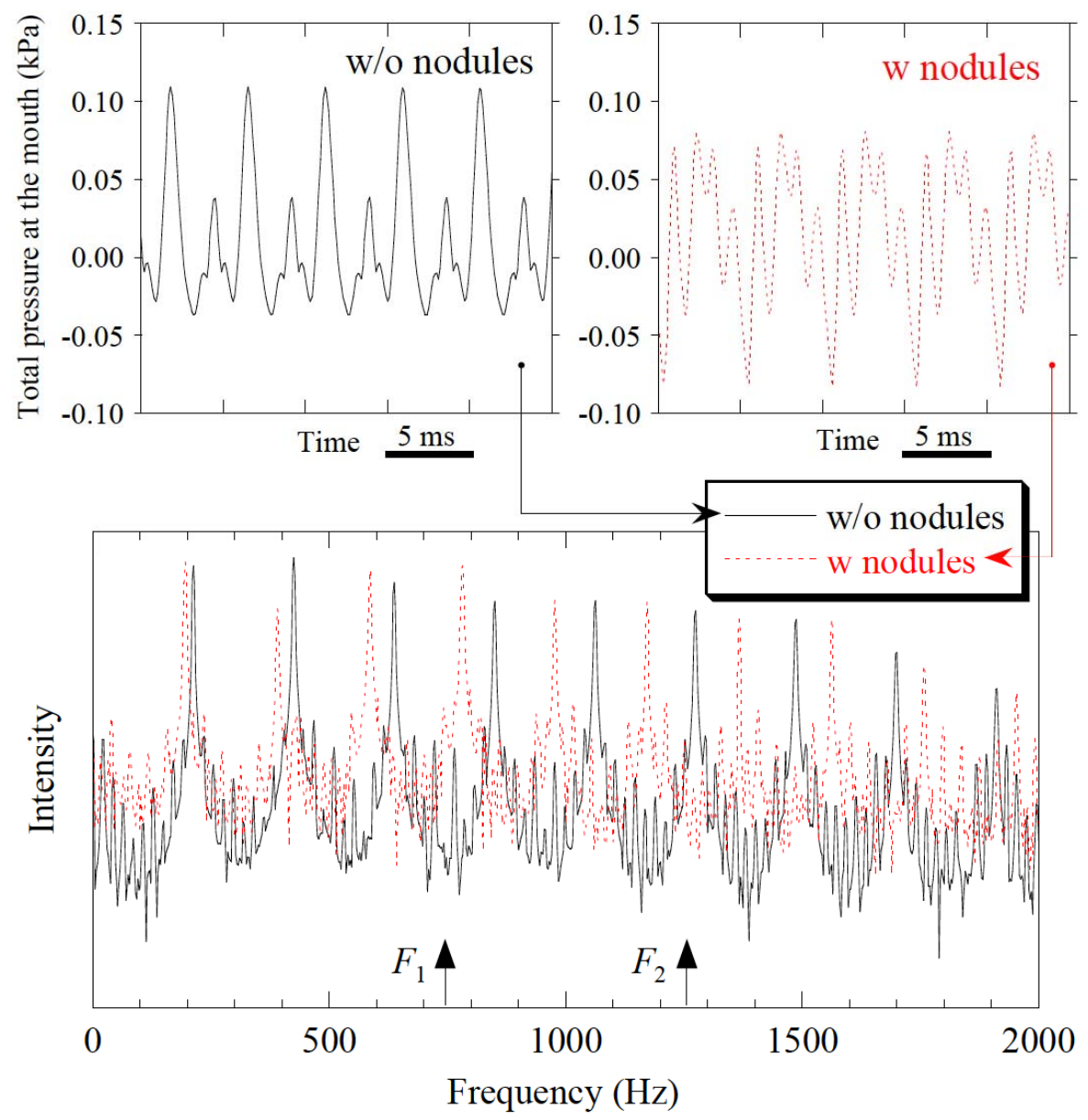

Figure 9. Sound waveform and spectrum of voice (total pressure at the mouth) with (w) or without (w/o) vocal nodules. $F_{1}$ and $F_{2}$ are the first and second formants determined by the geometry of the vocal tract shown in Figure 2 [22]. 


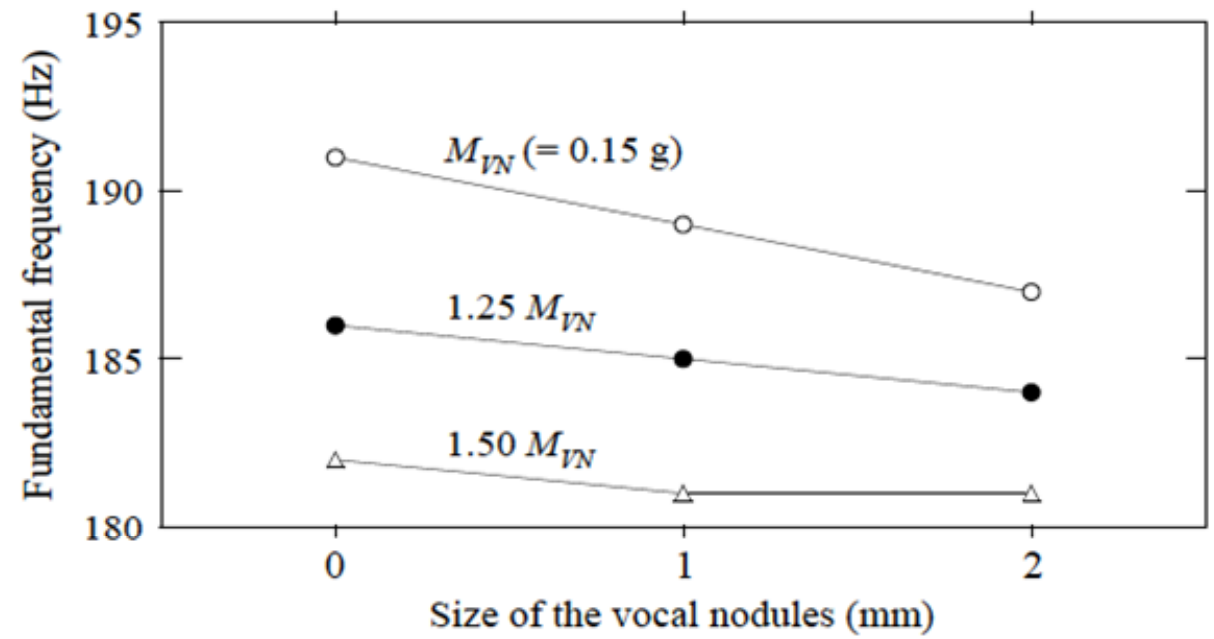

(a)

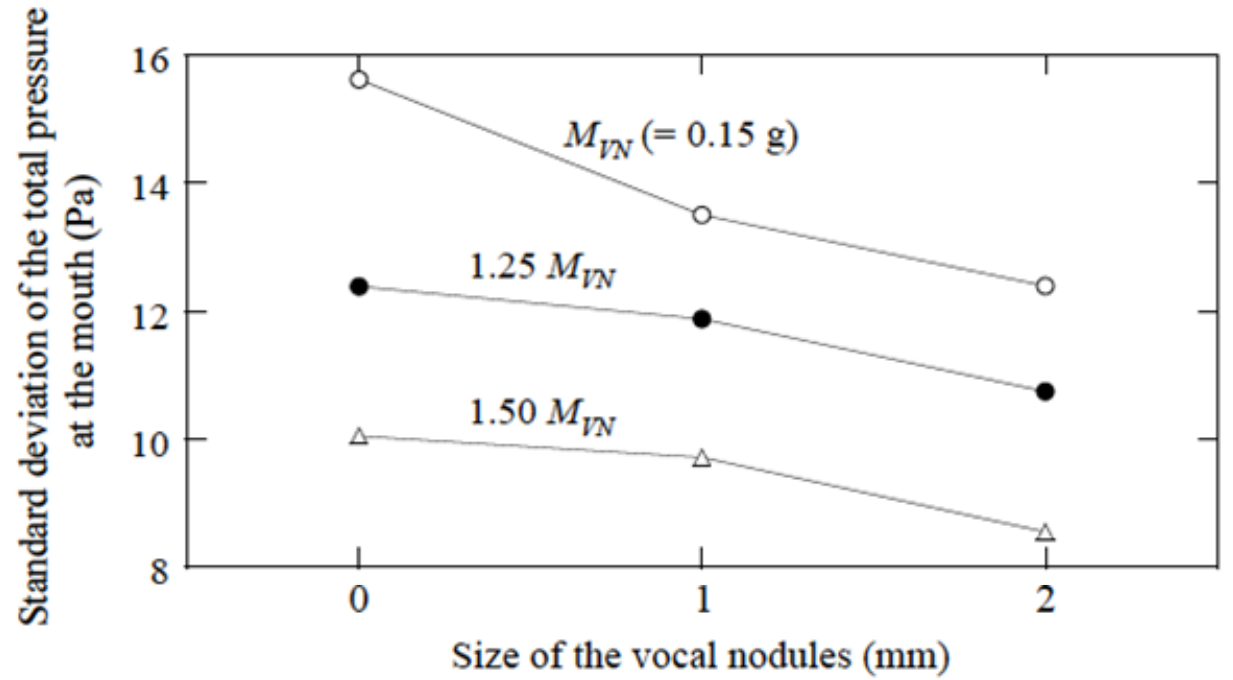

(b)

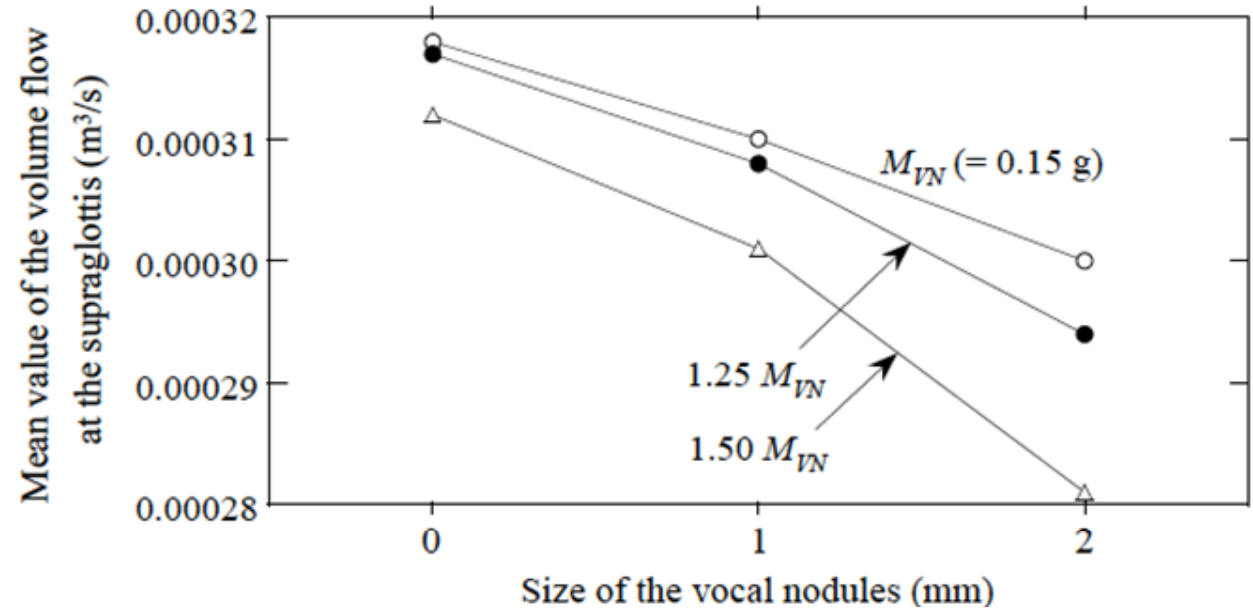

(c)

Figure 10. Effects of the vocal nodules on voice quality. The size of the vocal nodules vs. fundamental frequency (a), standard deviation of the total pressure at the mouth (corresponding to loudness of the voice) (b), and mean value of the volume flow at the supraglottis $(X=10.4 \mathrm{~mm})(\mathrm{c})$ as a function of their masses. 
The fundamental frequency was the lowest when the nodules were located at the center of fully adducted vocal fold length (Figure 11(d)). PTP, which can vary as a function of the fundamental frequency of voice, was greatest in the same nodule position (Figure 11(c)). As the nodules moved to the edge of the vocal fold length, the freely movable portion increased, allowing an easier onset of phonation (i.e., smaller PTP; Figure 11(c)) and a closer fluid-structure interaction (i.e., higher fundamental frequency; Figure 11(d)) [15]. Thus, the close association between the vocal fold motion and the voice frequency as well as the ease of phonation was influenced by the position of the vocal nodules. The negative correlation of Figures 11(c) and (d) shown in this model contradicts an analytical result previously reported [16] which states that PTP increases with the fundamental frequency. In previous findings, PTP increased with tissue stiffness or mass but decreased with the fluid-structure interactive geometric dimension (Equation (24)) [16]. However, this can be reconciled by noting that the fundamental frequency elevation that caused a rise in

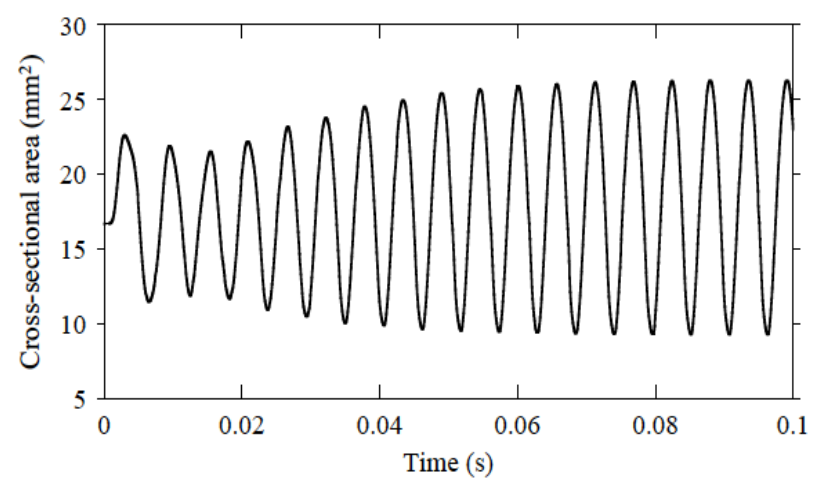

(a)

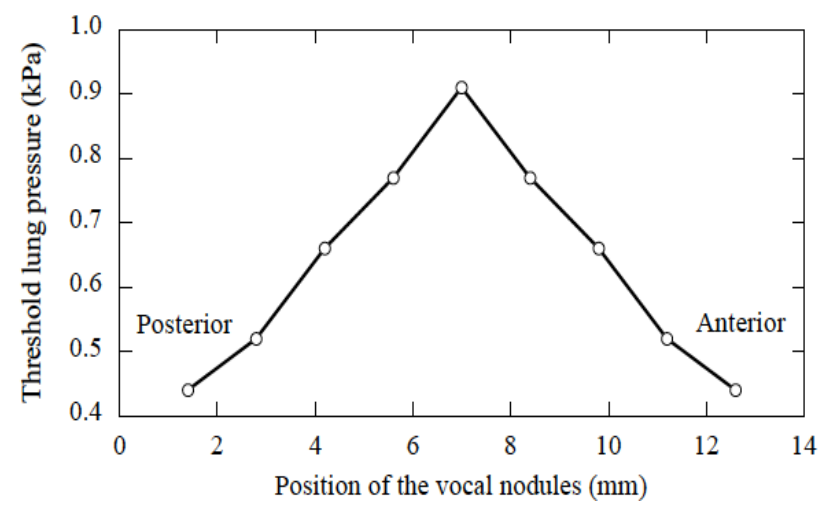

(c)
PTP was because of increase in tissue stiffness, without change in the mass or the interactive dimension.

The present model considers the three-dimensional geometry of the vocal folds and functional ability of incorporating active (muscular) and passive (ligamentous) stresses in the layered structure. Thus, clear relationships between the actual laryngeal structure and the model are observed, potentially allowing for more sophisticated simulation of disease conditions such as recurrent paralysis and vocal polyps. However, the model is not without limitations. In particular, initial glottal geometry in Equations (1) and (2) is determined independent of muscle activation. TA muscle activation was considered only for evaluating the tissue restoring force in Equation (13). The CT muscle activation was only implied for determination of the prestrains in Equations (8) and (11). In vivo, these muscle activations not only regulate the restoring force and prestrain but also determine the prephonatory positions of the laryngeal cartilages and vocal fold geometry, as discussed elsewhere [19-21]. Thus, the incorporation of muscle-dependent regulation of the prephonatory structure may be a desirable future direction.

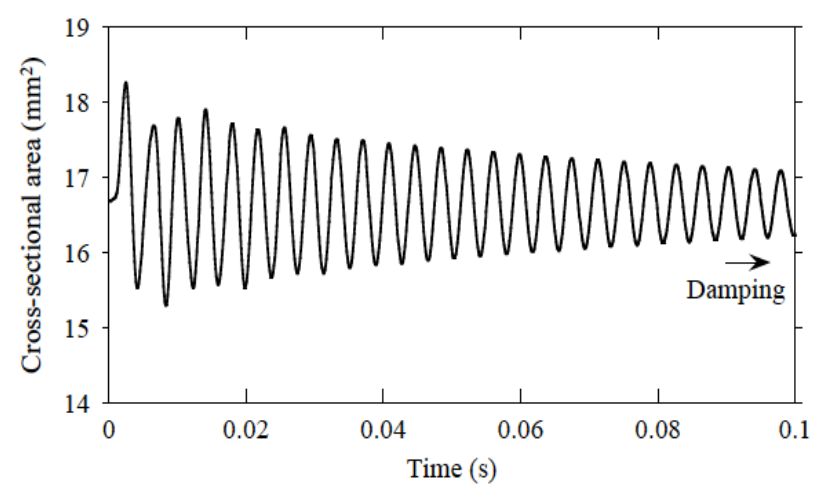

(b)

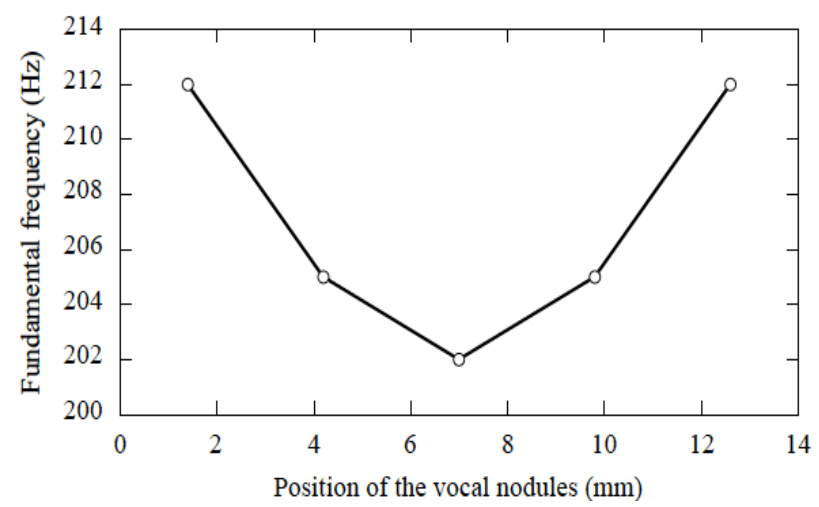

(d)

Figure 11. Effects of the vocal nodules on phonation efficiency. (a, b) Time course of cross-sectional area of the glottis at different lung pressures. Limit cycles are established at a high lung pressure $P_{l s}(a)$, while the oscillations gradually attenuate at low $P_{I s}(b)$. (c, d) Phonation threshold lung pressure and fundamental frequency of voice have an inverted v-letter and a v-letter curve, respectively, with respect to the position of the vocal nodules along the vocal fold length. 
In summary, we have developed a continuum-based numerical model for the simulation of human phonation with diseased vocal fold tissue. Here, we focused on the effect of varying localized bilateral masses to approximate the vocal nodules. The result shows that the presence of the nodules lowers the voice fundamental frequency and intensity. Phonation efficiency is most strongly decreased when the nodules are close to the center of the vocal fold length, where they prevent the fluid-structure interactions responsible for source sound production. These results suggest that phonation simulation using the newly developed model may be useful for indentifying the etiology of voice disorders.

\section{Acknowledgements}

The authors thank Yuji Matsuzaki and Tadashige Ikeda for providing advice on study design and Seiichi Washio and Satoshi Takahashi for discussion. SD gratefully acknowledges the support from the Japan Science and Technology Agency. This work was supported in part by Grant-in-Aid from the Ministry of Education, Culture, Sports, Science, and Technology of Japan (\#19700382 to $\mathrm{SD})$.

\section{References}

[1] K. Ishizaka and J. L. Flanagan, "Synthesis of Voiced Sounds from a Two-Mass Model of the Vocal Cords," Bell System Technical Journal, Vol. 51, No. 6, 1972, pp. 1233-1268.

[2] M. Hirano, "Morphological Structure of the Vocal Cord as a Vibrator and Its Variations," Folia Phoniatrica et Logopaedica, Vol. 26, No. 2, 1974, pp. 89-94. doi:10.1159/000263771

[3] B. H. Story and I. R. Titze, "Voice Simulation with a Body-Cover Model of the Vocal Folds," Journal of the Acoustical Society of America, Vol. 97, No. 2, 1995, pp. 1249-1260. doi:10.1121/1.412234

[4] T. Ikeda, Y. Matsuzaki and T. Aomatsu, "A Numerical Analysis of Phonation Using a Two-Dimensional Flexible Channel Model of the Vocal Folds," Journal of Biomechanical Engineering, Vol. 123, No. 6, 2001, pp. 571579. doi:10.1115/1.1408939

[5] C. Tao, J. J. Jiang and Y. Zhang, "Simulation of Vocal Fold Impact Pressures with a Self-Oscillating Finite-Element Model," Journal of the Acoustical Society of America, Vol. 119, No. 6, 2006, pp. 3987-3994. doi:10.1121/1.2197798

[6] S. Deguchi, Y. Matsuzaki and T. Ikeda, "Numerical Analysis of Effects of Transglottal Pressure Change on Fundamental Frequency of Phonation," Annals of Otology, Rhinology and Laryngology, Vol. 116, No. 2, 2007, pp. 128-134.

[7] I. T. Tokuda, J. Horácek, J. G. Svec and H. Herzel,
"Comparison of Biomechanical Modeling of Register Transitions and Voice Instabilities with Excised Larynx Experiments," Journal of the Acoustical Society of America, Vol. 122, No. 1, 2007, pp. 519-531. doi:10.1121/1.2741210

[8] X. Zheng, S. Bielamowicz, H. Luo and R. Mittal, "A Computational Study of the Effect of False Vocal Folds on Glottal Flow and Vocal Fold Vibration during Phonation," Annals of Biomedical Engineering, Vol. 37, No. 3, 2009, pp. 625-642. doi:10.1007/s10439-008-9630-9

[9] H. Hirose, "Clinical Aspects of Voice Disorders," Interuna Publishers, Tokyo, 1998, p. 173.

[10] L. Li, H. Saigusa, Y. Nakazawa, T. Nakamura, T. Komachi, S. Yamaguchi, A. Liu, Y. Sugisaki, E. Shinya and H. Shen, "A Pathological Study of Bamboo Nodule of the Vocal Fold," Journal of Voice, Vol. 24, No. 6, 2010, pp. 738-741. doi:10.1016/j.jvoice.2009.06.003

[11] D. Wong, M. R. Ito and N. B. Cox, "Observation of Perturbations in a Lumped-Element Model of the Vocal Folds with Application to Some Pathological Cases," Journal of the Acoustical Society of America, Vol. 89, No. 1, 1991, pp. 383-394. doi:10.1121/1.400472

[12] F. Alipour, D. A. Berry and I. R. Titze, "A Finite Element Model of Vocal-Fold Vibration," Journal of the Acoustical Society of America, Vol. 108, No. 6, 2000, pp. 3003-3012. doi:10.1121/1.1324678

[13] I. R. Titze and T. Riede, "A Cervid Vocal Fold Model Suggests Greater Glottal Efficiency in Calling at High Frequencies," PLoS Computational Biology, Vol. 6, No. 3, 2010, e1000897. doi:10.1371/journal.pcbi.1000897

[14] A. Yang, J. Lohscheller, D. A. Berry, S. Becker, U. Eysholdt, D. Voigt and M. Döllinger, "Biomechanical Modeling of the Three-Dimensional Aspects of Human Vocal Fold Dynamics," Journal of the Acoustical Society of America, Vol. 127, No. 2, 2010, pp. 1014-1031. doi: $10.1121 / 1.3277165$

[15] S. Deguchi and T. Hyakutake, "Theoretical Consideration of the Flow Behavior in Oscillating Vocal Fold," Journal of Biomechanics, Vol. 42, No. 7, 2009, pp. 824-829. doi:10.1016/j.jbiomech.2009.01.027

[16] S. Deguchi, "Mechanism of and Threshold Biomechanical Conditions for Falsetto Voice Onset," PLoS One, Vol. 6, No. 3, 2011, e17503. doi:10.1371/journal.pone.0017503

[17] O. Fujimura, "Body-Cover Theory of the Vocal Fold and Its Phonetic Implications," In: K. Stevens and M. Hirano, Eds., Vocal Fold Physiology, University of Tokyo Press, Tokyo, 1981, pp. 271-288.

[18] M. Hirano, K. Kiyokawa and S. Kurita, "Laryngeal Muscles and Glottic Shaping," In: O. Fujimura, Ed., Vocal Physiology, Mechanisms and Functions, Raven Press, New York, 1988, pp. 49-65.

[19] I. R. Titze, J. J. Jiang and D. G. Druker, "Preliminaries to the Body-Cover Theory of Pitch Control," Journal of Voice, Vol. 1, No. 4, 1988, pp. 314-319. doi:10.1016/S0892-1997(88)80004-3

[20] I. R. Titze, E. S. Luschei and M. Hirano, "The Role of the 
Thyroarytenoid Muscle in Regulation of Fundamental Frequency," Journal of Voice, Vol. 3, No. 3, 1989, pp. 213-224. doi:10.1016/S0892-1997(89)80003-7

[21] S. Deguchi, Y. Kawahara and S. Takahashi, "Cooperative Regulation of Vocal Fold Morphology and Stress by the Cricothyroid and Thyroarytenoid Muscles," Journal of Voice, in press.

[22] T. Baer, J. C. Gore, L. C. Gracco and P. W. Nye, "Analysis of Vocal Tract Shape and Dimensions Using Magnetic Resonance Imaging: Vowels," Journal of the Acoustical Society of America, Vol. 90, No. 2, 1991, pp. 799-828. doi:10.1121/1.401949

[23] G. R. Farley, "A Quantitative Model of Voice F0 Control," Journal of the Acoustical Society of America, Vol. 95, No. 2, 1994, pp. 1017-1029. doi:10.1121/1.408465

[24] T. Ikeda and Y. Matsuzaki, "A One-Dimensional Unsteady Separable and Reattachable Flow Model for Col- lapsible Tube-Flow Analysis," Journal of Biomechanical Engineering, Vol. 121, No. 2, 1999, pp. 153-159. doi:10.1115/1.2835097

[25] S. Deguchi, Y. Miyake, Y. Tamura and S. Washio, "Wavelike Motion of a Mechanical Vocal Fold Model at the Onset of Self-Excited Oscillation," Journal of Biomechanical Science and Engineering, Vol. 1, No. 1, 2006, pp. 246-255. doi:10.1299/jbse.1.246

[26] I. R. Titze, "The Physics of Small-Amplitude Oscillation of the Vocal Folds," Journal of the Acoustical Society of America, Vol. 83, No. 4, 1988, pp. 1536-1552. doi:10.1121/1.395910

[27] J. C. Lucero and L. L. Koenig, "On the Relation between the Phonation Threshold Lung Pressure and the Oscillation Frequency of the Vocal Folds," Journal of the Acoustical Society of America, Vol. 121, No. 6, 2007, pp. 3280-3283. doi:10.1121/1.2722210 